\title{
Learning of monocular information facilitates breakthrough to awareness during interocular suppression
}

\author{
Tommaso Mastropasqua • Peter U. Tse - Massimo Turatto
}

Published online: 27 February 2015

(C) The Psychonomic Society, Inc. 2015

\begin{abstract}
Continuous flash suppression (CFS) is a potent method of inducing binocular rivalry, wherein a rapid succession of high-contrast images presented to one eye effectively blocks from awareness a low-contrast image presented to the other eye. Here we addressed whether the contents of the suppressed image can break through to awareness with extended CFS exposure. On 2/3 of the trials, we presented a faint bar (the target) to the nondominant eye while a high-contrast flickering Mondrian (the mask) was displayed to the dominant eye. Participants were first asked to report whether the target had broken through the CFS mask. Furthermore, on targetpresent trials, the participants were then asked to guess whether the target had appeared above or below the fixation point. In Experiment 1, the target was presented with a fixed orientation for four blocks of trials, whereas in the fifth block, the target could also have the orthogonal orientation. In Experiment 2, the target was always presented with a fixed orientation, but in the fifth block, unbeknownst to participants, the target and the mask were swapped across the eyes. We found that awareness of the target rapidly improved with training in both experiments. However, whereas Experiment 1 revealed that the improvement largely generalized across stimulus orientations, Experiment 2 showed that the effect of practice was eye-specific. The results suggest that increased breakthrough with training was due to a monocular form of learning. Finally, a control experiment was conducted to exclude the possibility
\end{abstract}

T. Mastropasqua $(\bowtie) \cdot$ M. Turatto

Center for Mind/Brain Sciences, University of Trento, Trento, Italy

e-mail: tommaso.mastropasqua@unitn.it

P. U. Tse

Department of Psychology and Brain Sciences, Dartmouth College, Hanover, New Hampshire

M. Turatto

Department of Psychology and Cognitive Sciences, University of

Trento, Trento, Italy that the monocular learning we reported could have been due to sensory adaptation caused by the masks.

Keywords Binocular vision · Rivalry $\cdot$ Bistable perception . Perceptual learning $\cdot$ Visual awareness

Binocular rivalry occurs when two dissimilar images are presented simultaneously, one to each of the two eyes. Typically, the two images do not merge together into a single, stable percept, but instead, conscious experience alternates between the two images (Wheatstone, 1838). This feature has made binocular rivalry a valuable and elegant tool to study visual awareness and isolate its corresponding neural correlates (Blake \& Logothetis, 2002; Tong, Meng, \& Blake, 2006). It has been suggested that rivaling perceptual experience might arise because of a stage of winner-take-all competition (Blake \& Logothetis, 2002), which can occur at multiple levels in the visual system. According to one view, competition occurs quite early in the visual system, between eye inputs (Blake, 1989), whereas others have argued that competition occurs at later stages, among stimulus representations, regardless of eye of origin (Leopold \& Logothetis, 1996). More recently, however, the two distinct views have been reconciled by hybrid models, in which competition is thought to take place at both early and late stages of analysis in the visual system (Freeman, 2005; Wilson, 2003). One of the most interesting questions about binocular rivalry is how the visual system selects, at any given moment, the dominant image. Although alternations in visual experience during binocular rivalry are not strongly under the observer's control, von Helmholtz (1866/1925) was among the first to note that voluntary top-down attention could modulate the competition during rivalry, by extending the duration of the temporarily conscious image. The exact nature of such modulation (enhancement of the dominant 
image vs. suppression of the nondominant image) is not clear, but more recent evidence has confirmed Helmholtz's initial observations, showing that top-down task-related attention can prolong the dominant state of a rivalry stimulus (Chong, Tadin, \& Blake, 2005; Ooi \& He, 1999; but see Meng \& Tong, 2004; Sasaki \& Gyoba, 2002).

Because practice can improve the capacity to detect and discriminate sensory input (Fahle \& Poggio, 2002; Frank, Reavis, Tse, \& Greenlee, 2014), we reasoned that with appropriate training, one might also learn to bring the contents of the suppressed image into awareness. Fortunately, in recent years the development of a new technique, called continuous flash suppression (CFS; Tsuchiya \& Koch, 2005), has overcome some of the limitations of binocular rivalry (Kim \& Blake, 2005; Tsuchiya, Koch, Gilroy, \& Blake, 2006), particularly the limitation imposed by the intrinsic unpredictability of the timing of switches in dominance. In CFS, a static low-contrast target stimulus is presented to one eye, while a high-contrast masking stimulus is continuously flashed into the other eye. As a result, conscious perception does not spontaneously and unpredictably alternate between the two competing images, as in binocular rivalry. Instead, the static low-contrast stimulus can remain largely invisible for long durations, making CFS an ideal tool to study the impact of invisible stimuli on different perceptual and cognitive processes (e.g., Fogelson, Kohler, Miller, Granger, \& Tse, 2014; Jiang, Costello, Fang, Huang, \& He, 2006; Tsuchiya \& Koch, 2005), as well as to address the functional organization and neural correlates of unconscious processing in the visual system (e.g., Almeida, Mahon, \& Caramazza, 2010; Fang \& He, 2005; Jiang \& He, 2006). However, other studies have also used CFS to measure the time it takes for an initially invisible target stimulus to win the competition with the high-contrast masking image and reach awareness. In this case, the contrast of the target stimulus is ramped up gradually to a value at which the target breaks through the mask, and the observers are asked to press a button as soon as the target becomes consciously visible (e.g., Jiang, Costello, \& He, 2007).

To address whether practice can enhance the likelihood that the suppressed image can enter visual awareness, we capitalized on the fact that, due to the stochastic nature of rivalry, even under CFS, the weaker (initially suppressed) image can, sometimes, locally break through the high-contrast flickering image. van Ee (2011), addressing local breakthrough of the suppressed image during binocular rivalry, showed that neuronal mechanisms of local adaptation in early visual monocular representations likely account for the switch in perceptual awareness across portions of the two eyes' images. If this is correct, any changes in breakthrough probability should occur because of changes in monocular circuits. This makes the prediction that switching the eye of target presentation should eliminate any increases in breakthrough probability that might have been observed during training prior to this eye switch.
Furthermore, recent studies have also documented effects of training on interocular suppression. For example, Paffen, Verstraten, and Vidnyànszky (2008) showed that the unattended task-irrelevant feature in a perceptual-learning task was more strongly suppressed than the task-relevant feature during a subsequent binocular rivalry task. Also, Ludwig, Sterzer, Kathmann, Franz, and Hesselmann (2013) have provided initial evidence suggesting that with training, the visibility of the suppressed image in a CFS paradigm can improve, although this was not the main goal of their study, which was instead aimed at finding perception-action dissociations in neurologically intact observers. The authors found that as the experiment unfolded, it was necessary to reduce the suppressed target's contrast to maintain full invisibility during CFS.

The present set of experiments was instead specifically designed to foster, through practice, the increased visibility of the suppressed target image during interocular suppression. To this end, we exploited the long duration of rivalrous suppression afforded by CFS. But instead of measuring the target breakthrough time, we recorded the rate of breakthrough events, which we treated as an index of consciousness of the weaker image. In addition, since one of the most debated issues about binocular rivalry and CFS is the stage(s) of processing at which the competition between the two images for access to awareness is resolved, we devised a paradigm that allowed us to address whether any training-induced improvements of target awareness was based on a learning process that operates at the level of the target representation or, conversely, at the level of an eye-specific representation.

In both experiments, participants were presented with a different image to each eye for approximately $3 \mathrm{~s}$ : A static, faint-gray bar, the target, was presented (on $2 / 3$ of the trials) on a uniform dark-gray background to the nondominant eye, whereas a continuously changing high-contrast grayscale Mondrian pattern, comprising the mask, was presented to the dominant eye (Fig. 1). To determine the dominant eye, we used the Porta test: We asked participants to align a finger with a distant point while keeping both eyes open. Then participants closed each eye in turn, and reported the eye for which the finger remained more aligned with the point (specifying the dominant eye), and the eye for which the finger shifted away from the point (specifying the nondominant eye).

On each trial, the first task was to report whether or not the target broke through the Mondrian mask to become consciously visible. We regarded this as the main task addressing visual awareness of the target, which we quantified as the rate of breakthrough events. However, since we also presented catch trials $(1 / 3$ of the total trials), in which the target was not shown, we had the possibility to control whether the measured rate of breakthrough events reflected a simple response bias. Furthermore, participants had to report, in a twoalternative forced choice (2AFC) task, the location (above or below the fixation point) of the target appearance. This was 


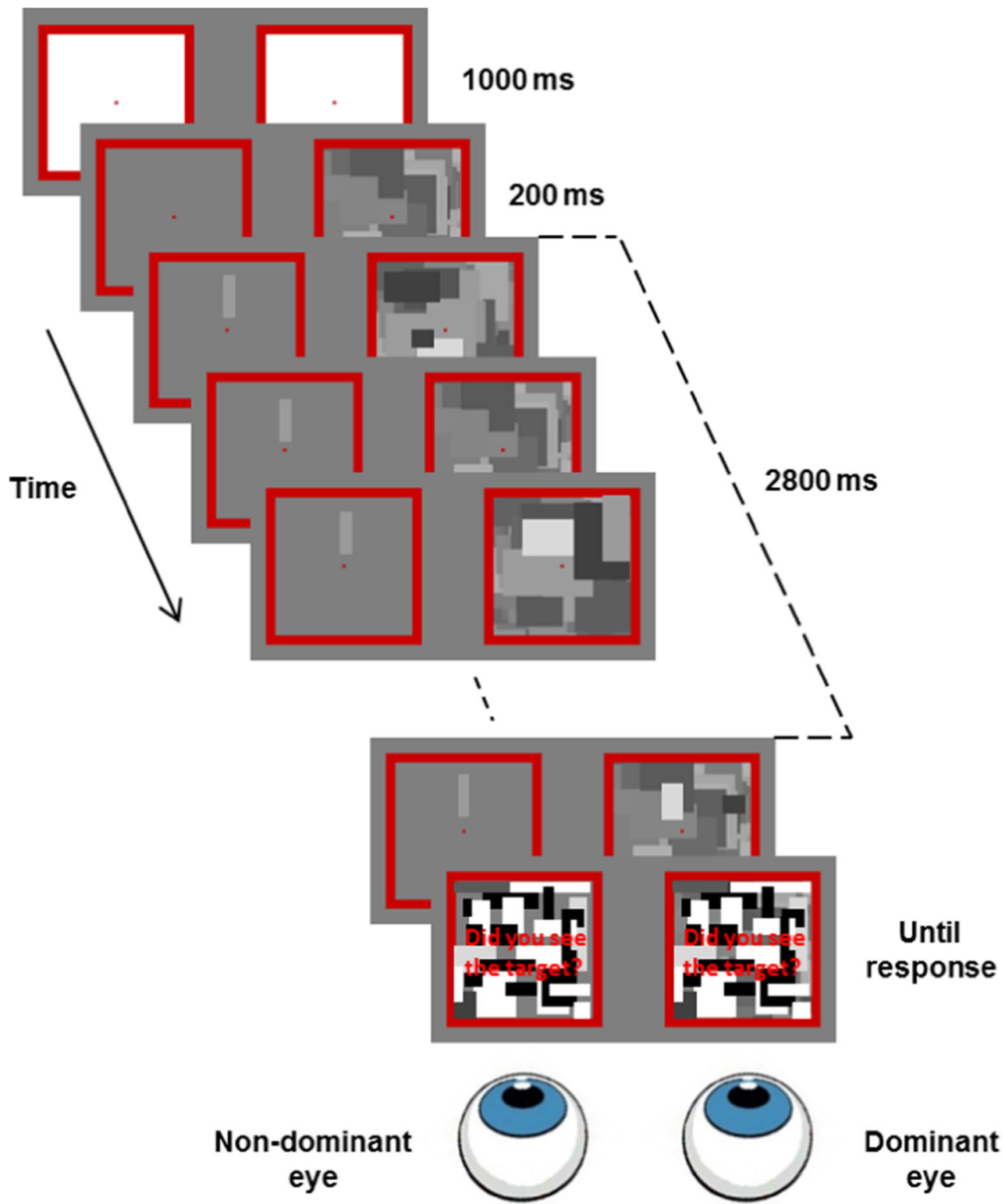

Fig. 1 Schematic representation of events in Experiments 1 and 2. During the training phase (Blocks 1-4), the dynamic mask (Mondrian patches) was presented to the dominant eye, and the static target to the nondominant eye. During the test phase (Block 5), in Experiment 1, the

done to ascertain whether the reported breakthrough events corresponded to genuine conscious perception of the target, when this was presented, and to ascertain whether there was unconscious processing for position even when there was no breakthrough. This second task allowed us to estimate each participant's sensitivity to the target's occurrences in the two designated locations. We then used the presence of sensitivity in the $2 \mathrm{AFC}$ task to quantify, for each participant, the reliability of the reported breakthrough events as an index of conscious perception of the target.

One may note that in the first task, the proportions of breakthrough events in the target-present trials and the target-absent target changed from vertical to horizontal (or vice versa) on half of the trials, whereas in Experiment 2, the target remained the same, but on half of the trials the target and the mask were swapped across the eyes

trials corresponded to hits and false alarms, in the terminology of signal detection theory (Green \& Swets, 1966). However, two reasons motivated us to analyze these two measures separately, instead of combining them into a single measure, $d^{\prime}$. First, CFS, as well as binocular rivalry, is generally recognized as a technique that can be used to directly address conscious vision. In particular, when a suppressed image breaks through the mask, its content is believed to be part of the observer's conscious experience. For this reason, and in agreement with previous studies on CFS using the breakthrough time as a measure of the moment on which conscious perception of the previously suppressed image arises, we used the 
proportion of trials in which the observers reported having experienced breakthrough as an index of target awareness. Second, the choice of $d^{\prime}$ as a measure of consciousness can be questioned, since $d^{\prime}$ is a measure of performance; changes in $d^{\prime}$ are not necessarily accompanied by any corresponding changes in conscious experience. Indeed, the blindsight phenomenon shows a remarkable dissociation between lack of awareness (no detection of the stimulus) and sensitivity (the capacity to discriminate some properties of the "unseen" stimulus). Hence, in line with previous studies using CFS, we adopted the strategy of considering breakthrough a subjective report of conscious target detection, and $d^{\prime}$ in the 2AFC task an objective measure of the participant's sensitivity.

\section{Experiment 1}

In this experiment, we addressed whether it is possible to increase awareness of the suppressed image with practice, and whether the improved awareness of the weaker image is due to changes in representations specific to the stimulus. This possibility has indeed been suggested by evidence showing that binocular rivalry can arise from competition between different object representations, regardless of the eye of origin (Kovács, Papathomas, Yang, \& Fehér, 1996; Logothetis, Leopold, \& Sheinberg, 1996). Hence, in the present experiment the target that participants had to detect, and localize when present, always had the same orientation (vertical or horizontal) in the first four blocks of trials, whereas in the fifth block, one or the other orientation was randomly presented on each trial. Because vertical and horizontal orientations activate largely distinct neural populations, at least in V1 (Hubel \& Wiesel, 1977), if any improvement in target awareness were to arise because training induced changes at the level of the stimulus representation in one such population, practicing in the first four blocks on one orientation should not transfer an increased tendency for breakthrough to the opposite orientation.

\section{Method}

Participants Eighteen undergraduate students from the University of Trento ( 15 females, three males; mean age $=$ 21 years) participated for course credits or monetary compensation. All of the participants had normal or corrected-tonormal vision and were unaware of the purpose of the experiment, which was carried out with the approval of the local research ethics committee. All volunteers gave informed consent prior to participation.

Stimuli and apparatus The experiment took place in a dimly illuminated and quiet psychophysics testing room. The visual stimuli were presented on a gamma-calibrated monitor (CRT, 19-in., 1,024 × 768, $100 \mathrm{~Hz}$ ). Participants rested their chin at a distance of $60 \mathrm{~cm}$ and perceived the stimuli through a custombuilt stereoscope that presented, via mirrors, different images to the two eyes. To promote and ensure stable binocular convergence, the mirrors were adjusted individually for each participant at the beginning of each block of trials, to assure that the identical images presented to the two eyes exactly overlapped. A program written using MATLAB and the Psychophysics Toolbox 3.8 (Brainard, 1997) managed stimulus presentation and data storage.

The target, presented to the nondominant eye, consisted of a gray bar $\left(1.6^{\circ}\right.$ in height, $0.4^{\circ}$ in width, contrast of $\left.10 \%\right)$, which was displayed against a darker background $\left(45 \mathrm{~cd} / \mathrm{m}^{2}\right)$. The mask, presented to the dominant eye, consisted of grayscale Mondrian patches. Each Mondrian patch was the result of 500 overlapping rectangles, randomly sized and filled with one of six possible grayscale levels (mean contrast of $40 \%$ ). The edges of each rectangle could vary between $1.3^{\circ}$ and $2.6^{\circ}$. On a given trial, the Mondrian patches changed at one of four different temporal frequencies: 10, 15, 20, and $50 \mathrm{~Hz}$. We adopted different frequencies instead of a fixed one because we wanted to be able to generalize our findings, regardless of the frequency used. To foreshadow the results, the use of different frequencies had no effect on the perceptual tasks.

The target and the mask appeared inside a red frame $\left(6.7^{\circ} \times\right.$ $6.7^{\circ}, 0.4^{\circ}$ in thickness $)$ with a central fixation dot $\left(0.2^{\circ}\right.$ diameter) in the corresponding eye. The distance between the central dots of the two eyes was $9.3^{\circ}$. The mirrors of the stereoscope were adjusted so that the two images were fused into a single image, such that the presented stimuli completely retinotopically overlapped.

Eye blinks were monitored using an eyetracker (EyeLink 1000 Desktop Mount, SR Research; 500-Hz sampling rate). If an eye blink was detected during the 3-s presentation of the stimuli, a warning message was presented, and the trial was aborted and immediately re-presented.

Procedure The present and the following experiment consisted of a single session that lasted about $1 \mathrm{~h}$. The session started with ten practice trials, followed by five experimental blocks of trials. The first four blocks served as training and contained 48 trials each; the last block served as the test and had double the number of trials (96). Prior to starting the practice trials, participants were informed that the target would be absent on some proportion of trials, and that, when present, it could have different orientations. Participants were shown a display on the computer screen depicting the two possible target orientations and positions (below and above the fixation point). They were also informed that their task was to report the appearance of the target in their perceptual experience, usually dominated by the mask.

To investigate whether any improved target awareness was specific to orientation, participants were first trained with a single target orientation (vertical or horizontal, counterbalanced 
across participants), and then were tested with both orientations in Block 5. Each of the four training blocks consisted of 16 target-absent and 32 target-present trials. On half of the targetpresent trials of each block, the target appeared above the fixation dot, whereas it appeared below the fixation dot on the remaining trials.

Each trial began with the presentation of two white-filled red frames for $1,000 \mathrm{~ms}$. Then, Mondrian patches were flashed at a given frequency for $3,000 \mathrm{~ms}$ to the dominant eye. If present, the target appeared in the nondominant eye for $2,800 \mathrm{~ms}$, starting with a delay of $200 \mathrm{~ms}$ from the onset of the Mondrians. Once the 3,000-ms sequence was complete, the Mondrian image presented in the last frame of the Mondrian mask sequence was shown at full contrast $(100 \%)$ in both eyes. A written sentence, superimposed on the Mondrian image, asked participants whether or not they had seen the target. Participants pressed the "b" key for a positive answer and the " $n$ " key for a negative answer with their left hand, without any time pressure. The same question was asked twice, because we wanted to be confident that the reported breakthrough corresponded to a genuine perceptual experience of the target. Therefore, the purpose of the second response was to give participants the opportunity to confirm the first one or, if necessary, to change their mind. However, the proportion of trials on which the two responses differed was negligible.

Finally, but only on target-present trials, participants were engaged in a $2 \mathrm{AFC}$ task in which they had to discriminate whether the target had appeared just above or below the fixation point. With their right hand, and without time pressure, participants pressed the " 2 " key to indicate that the target had appeared above the fixation dot, or the " 5 " key to indicate that it had appeared below. Incorrect responses to the location discrimination task were signaled using auditory feedback.

Once participants had entered their responses, the two fullcontrast Mondrians were replaced by two white backgrounds, at full contrast, for $1 \mathrm{~s}$.

\section{Results and discussion}

The first analysis addressed whether the conscious visibility of the target increased with training. The $2 \%$ of trials on which a breakthrough event was not consistently reported (in the two responses to the single question whether participants had experienced breakthrough) were discarded from further analysis. Then, the proportions of breakthrough events for the trained target were entered into a repeated measures analysis of variance (ANOVA), with Block and Frequency as factors. We observed a robust increase in the proportions of breakthrough events across blocks (Block $1, M=.54$; Block 2, $M=.60$; Block 3, $M=.63$; Block 4, $M=.63$; Block 5, $M=.66$ ), as was attested by the significant main effect of block, $F(4,68)=$ $3.038, p=.023, \eta^{2}=.152$. In contrast, neither the main effect of frequency, $F(3,51)=0.571, p=.636, \eta^{2}=.033$, nor the Block $\times$ Frequency interaction, $F(12,204)=1.174, p=.304$, $\eta^{2}=.065$, was significant. Note, however, that due to the limited number of trials (eight) in each cell of the Frequency $\times$ Block interaction, any conclusion about the role of frequency should be regarded with caution.

We interpret the increase of breakthrough events across blocks as evidence of improved target awareness or of the reduced efficacy of the mask. One cannot argue that this result arose from a change in decision-making bias, such that participants became more prone to report target presence across subsequent blocks, regardless of any change in conscious experience of the target; if this were the case, we should have also observed an increase in the proportion of target detection events in target-absent trials. Because the analysis of targetabsent trials revealed that the proportions of target detection events significantly decreased across blocks (Block $1, M=$ .39 ; Block 2, $M=.32$; Block 3, $M=.27$; Block 4, $M=.24$; Block $5, M=.21), F(4,76)=8.692, p<.001, \eta^{2}=.314$, we can rule out the possibility that the increment in breakthrough events in target-present trials was due to a response bias. On the contrary, we can conclude that the pattern of breakthrough events across blocks represents an effective improvement in target conscious perception. One should also note that an increment (across blocks) in the proportions of breakthrough events in target-present trials, together with a corresponding decrement of the same responses in target-absent trials, can only be explained by an improvement in target sensitivity $\left(d^{\prime}\right)$ in the detection task.

Furthermore, in order to set a more stringent criterion for what counted as training-induced improvements in target awareness, we only considered breakthrough events when participants also showed a significant sensitivity in discriminating the target location. We reasoned that if a participant reported having seen the target break through the mask, but could not report where the stimulus appeared (above or below the fixation point), this cast serious doubt on the claim that the target had been consciously perceived. Hence, since we were interested in studying whether target awareness can improve with practice during CFS, we reanalyzed the proportion of breakthrough events in target-present trials by excluding those participants who showed no sensitivity $\left(d^{\prime}\right)$ in the target localization task, since these participants presumably had no awareness of the target. It is important to note that by applying this criterion, we did not select those participants who showed learning in the 2AFC task, but only those who showed sensitivity, which by itself does not guarantee learning as a consequence of training. In principle, indeed, sensitivity could also remain unaffected by the relatively modest amount of practice involved in our experiments.

To assess whether $d^{\prime}$ in the 2AFC task was significantly different from zero, we used a bootstrap procedure in which, for each participant, we estimated the $95 \%$ confidence 
interval (CI) of $d^{\prime}$, sampling with replacement from the observed data including all blocks (this sampling was repeated 10,000 times). By definition, a $d^{\prime}$ value was significantly different from zero if the relative CI did not contain zero. The application of this null-sensitivity criterion removed seven out of 18 participants, who showed no sign of sensitivity $\left(d^{\prime}=0\right)$. It is not surprising that a consistent number of participants showed no sensitivity in the $2 \mathrm{AFC}$ task during CFS, since this technique has been shown to be very effective in causing the complete invisibility of the target (e.g., Fang \& He, 2005; Tsuchiya \& Koch, 2005). Evidently, for some of our participants the physical strength of the flickering Mondrian, as compared to that of the target, was strong enough to mask the target throughout the experimental session.

For the remaining 11 participants, who showed a $d$ ' significantly larger than zero in the target localization task, the proportions of breakthrough events for the trained target were entered again into an ANOVA with the same factors as before. The results confirmed a robust effect of learning, attested by a significant main effect of block, which was the only factor that reached significance, $F(4,40)=7.310, p<.001, \eta^{2}=.422$ (Fig. 2a). Also for these participants, no indication of response bias was found when we analyzed the pattern of target detection in target-absent trials, since the proportion of reported breakthrough events significantly decreased across blocks (Block 1, $M=.32$; Block 2, $M=.28$; Block 3, $M=.21$; Block $4, M=.16$; Block $5, M=.15), F(4,40)=4.416, p=$ $.005, \eta^{2}=.306$.

To evaluate whether the improved visibility of the target achieved with training involved changes in the stimulusspecific representations, we compared, in the last block, the proportion of breakthrough events between trials on which we presented the trained target (e.g., vertical) with that on which we presented the new target (e.g., horizontal). Pairwise comparisons ( $t$ tests) showed that, as compared to the trained target, the proportion of breakthrough events slightly but

A

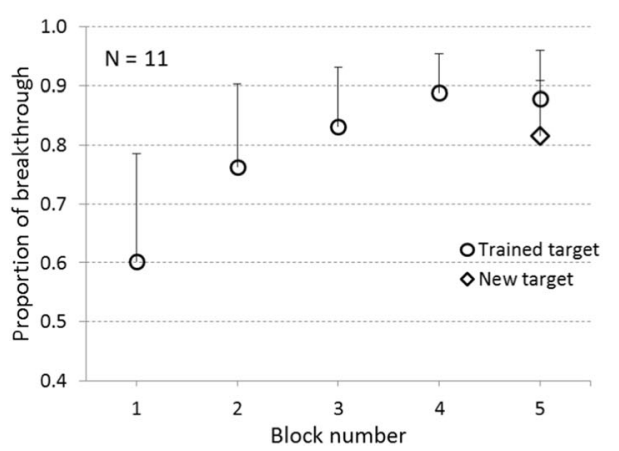

Fig. 2 a Proportions of breakthrough events for those participants showing sensitivity $\left(d^{\prime}\right)$ in the target localization task. The conscious visibility of the trained target increased across blocks. However, target visibility slightly decreased when the target orientation was changed (see Block 5). b Performance in the two-alternative forced choice (2AFC) task, plotted as a function of blocks separately for breakthrough and no- significantly decreased for the new target orientation, $t(10)=$ $2.84, p=.018, r^{2}=.45$, although a large part of the achieved improvement was retained, as revealed by the difference in breakthrough events between the new target and the trained target in the first block, $t(10)=2.79, p=.019, r^{2}=.44$ (Fig. 2a).

Furthermore, performance in the $2 \mathrm{AFC}$ task also improved across blocks on breakthrough trials, $F(4,40)=8.616, p<$ $.001, \eta^{2}=.463$ (Fig. 2b), thus confirming that the increase in breakthrough events corresponded to a genuine improvement in conscious target perception. It was not possible to analyze the performance for no-breakthrough trials, since many participants had cells of the design with no data. However, when data were collapsed across blocks, participants' discriminative capacity was higher in breakthrough $\left(d^{\prime}=2.35, \mathrm{CI}=[1.77\right.$, 2.93]) than in no-breakthrough $\left(d^{\prime}=0.11, \mathrm{CI}=[-0.18,0.39]\right)$ trials, $t(10)=6.86, p<.001, r^{2}=.82$, and was also significantly higher than 0 in the former, $t(10)=9.05, p<.001, r^{2}=$ .89 , but not in the latter, $t(10)=0.84, p=.419, r^{2}=.07$, condition.

Finally, it could be argued that our exclusion criterion was too stringent, because it was based on participants' performance in the 2AFC task, which could be more difficult than the simple detection task. In other words, we could not exclude the possibility that detecting the breakthrough of the target could be easier than discriminating its position. Therefore, we might have excluded a large number of participants who actually achieved a high performance level in the detection task. To rule out this possibility, we plotted the $d$ 's of each participant (computed considering all blocks) in both tasks. As is depicted in Fig. 3a, with the exception of one participant (indicated by the open symbol), all of the excluded participants who showed no sensitivity in the 2AFC task also showed no sensitivity in the target detection task (to calculate whether $d^{\prime}$ in the yes/no task was different from zero, we applied the same bootstrap procedure used for the 2AFC task).

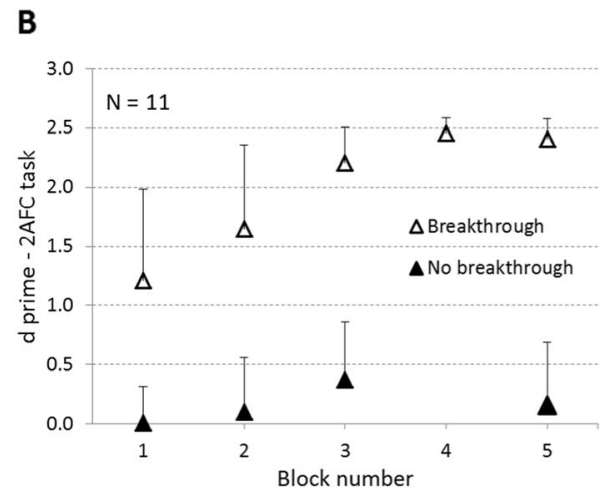

breakthrough trials. Sensitivity was significantly higher when the target was consciously perceived, as compared to when it did not break through the mask. The data point in the fourth block is missing because there were not enough data to calculate the $d^{\prime}$ value in the 2AFC task, given a "no" response in the yes/no task. Error bars represent $95 \%$ confidence intervals 


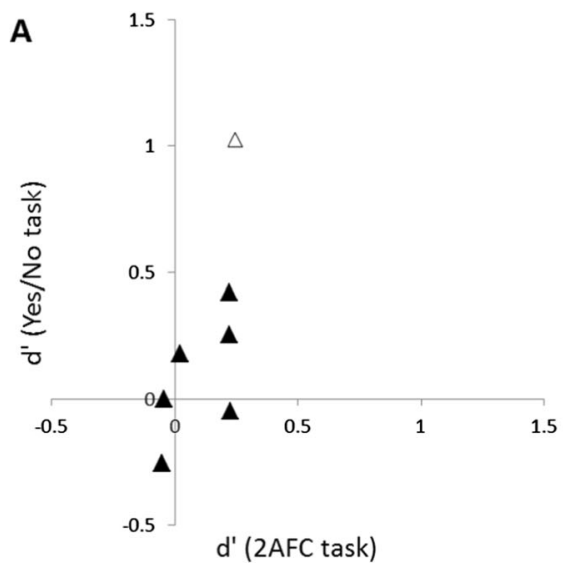

Fig. 3 Performance in the two-alternative forced choice (2AFC) and yes/ no tasks for those participants discarded on the basis of our exclusion criteria in (a) Experiment 1 and (b) Experiment 2. In both panels, the open

In summary, there were two key results of this first experiment. First, in a CFS paradigm, participants rapidly learned to bring the content of the suppressed visual representation into awareness with relatively modest training; second, the beneficial effect of training did not seem to take place at the level of the specific stimulus representation, because what was gained with practice in terms of improved awareness for a given (e.g., vertical) target orientation was almost completely retained when the orientation of the target was changed to the orthogonal orientation. The small drop in performance observed in trials on which the target orientation changed could also be explained by noticing that during training, participants were presented with a fixed target orientation. As a consequence, participants may have developed an expectation for the trained target orientation, which might have facilitated its breakthrough, relative to the new orientation.

\section{Experiment 2}

In the second experiment, we addressed two issues. Our first aim was to replicate the effect of training on visual awareness we had found in Experiment 1. Secondly, we designed Experiment 2 with the goal of elucidating at which stage of analysis training affected participants' awareness of the weaker image. Having reasonably ruled out that learning occurred mainly at the level of a stimulus's representation - such as, say, among the orientation-tuned units in $\mathrm{V} 1$ - we then tested the alternative possibility - namely, that the practice-induced enhancements of breakthrough to awareness for the suppressed image were due to a learning process that can operate over eye-specific information. In particular, in this experiment we tested the possibility that participants improved their awareness of the suppressed target by altering monocular retinotopic-specific representations.

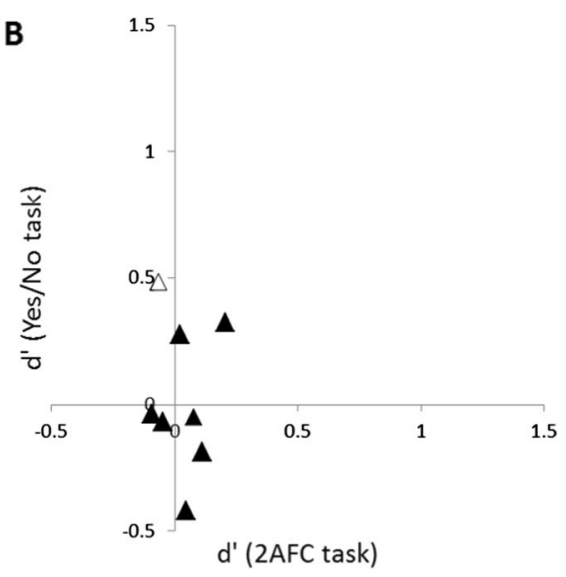

symbol indicates the only participant who showed $d^{\prime}>0$ in the yes/no task, despite a $d^{\prime}$ not different from 0 in the $2 \mathrm{AFC}$ task

Method

Participants Twenty-one undergraduate students from the University of Trento $(17$ females, four males; mean age $=$ 20 years) participated for course credit or monetary compensation. All of the participants had normal or corrected-tonormal vision and were unaware of the purpose of the study, which was carried out with the approval of the local research ethics committee. All volunteers gave informed consent prior to participation.

Stimuli and apparatus These were the same as in Experiment 1.

Procedure This was also as in Experiment 1, but with the following exceptions: First, the target had the same orientation (vertical) in all blocks of trials; second, whereas in the first four blocks of training the target was always presented to the nondominant eye, and the mask to the dominant eye, in the last (testing) block, unbeknownst to participants, the target and mask were swapped across eyes on half of the trials.

\section{Results and discussion}

First, we calculated the proportion of trials on which a breakthrough event was not consistently reported in the two responses to the question asking whether there had been a breakthrough event, and these trials $(1 \%)$ were discarded from further analysis. Then, the proportions of breakthrough events for the trained target were entered into a repeated measures ANOVA, with Block and Frequency as factors. We replicated the increase in the proportion of breakthrough events across blocks (Block 1, $M=.60$; Block 2, $M=.64$; Block 3, $M=.70$; Block 4, $M=.73$; Block 5, $M=.70$ ), as revealed by the significant main effect of block, $F(4,80)=3.395, p=.013$, $\eta^{2}=.145$. In contrast, neither the main effect of frequency, 
$F(3,60)=0.621, p=.604, \eta^{2}=.030$, nor the Block $\times$ Frequency interaction, $F(12,240)=0.485, p=.922, \eta^{2}=$ .024 , was significant. As was already discussed in Experiment 1, the null effect of frequency should be regarded with caution, because of the low number of elements in each frequency cell of the design.

Again, to exclude the contribution of any response bias, we also analyzed the pattern of breakthrough events for targetabsent trials. As in Experiment 1, the results were clear and showed that the proportion of target detection events significantly decreased across blocks (Block $1, M=.37$; Block 2, $M=.34$; Block 3, $M=.31$; Block 4, $M=.28$; Block 5, $M=$ $.28), F(4,80)=3.494, p=.011, \eta^{2}=.149$, thus ruling out any explanation based on response bias.

We then applied the same null-sensitivity criterion used in Experiment 1 to remove those participants who showed no sensitivity in the target localization task. This led to the removal of eight out of the 21 participants. The proportions of breakthrough events for the remaining 13 participants were entered into a further ANOVA with the same factors as before; the results confirmed that awareness of the target increased with practice, as revealed by the significant factor Block, $F(4,48)=5.740, p=.001, \eta^{2}=.324$ (Fig. 4). As before, no evidence of response bias emerged in this subset of participants, since the proportion of reported breakthrough events significantly decreased with training in target-absent trials (Block 1, $M=.29$; Block 2, $M=.21$; Block 3, $M=.20$; Block $4, M=.15$; Block $5, M=.16), F(4,48)=6.511$, $p<.001, \eta^{2}=.352$.

To evaluate whether the improved visibility of the target across blocks involved eye-specific representations, we compared, in the last block, the proportion of breakthrough events for trials on which the target was presented to the same eye to which it had been shown during training, with that on which the target was presented to the other eye. Pairwise

A

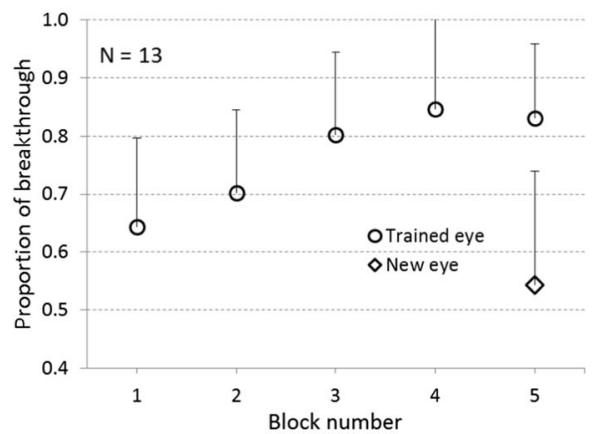

Fig. 4 a Proportions of breakthrough events for those participants showing sensitivity $\left(d^{\prime}\right)$ in the target localization task. Conscious visibility of the trained target increased across blocks. However, target visibility dramatically decreased when the eyes were swapped (see Block 5). b Performance in the two-alternative forced choice task, plotted as a comparisons ( $t$ tests) showed that in trials on which the eyes of the target and the mask were switched, breakthrough to awareness was dramatically reduced, $t(12)=3.50, p=.004$, $r^{2}=.51$. In addition, the degree of target awareness in the neweye trials returned to the level of the first block, $t(12)=0.98, p$ $=.347, r^{2}=.07$ (Fig. 4a). This pattern of results gives strong support to the conclusion that learning involved monocular retinotopic-specific information.

As in Experiment 1, performance in the 2AFC task on breakthrough trials also improved across blocks (Fig. 4b), $F(4,48)=9.725, p<.001, \eta^{2}=.448$, thus confirming the genuine enhancement in conscious target perception indicated by the breakthrough events analysis. Again, due to a lack of data, it was not possible to analyze the performance for nobreakthrough trials. However, when data were collapsed across blocks, participants' discriminative capacity was higher in breakthrough $\left(d^{\prime}=2.42, \mathrm{CI}=[1.90,2.95]\right)$ than in no-breakthrough $\left(d^{\prime}=0.20, \mathrm{CI}=[-0.13\right.$, $0.54]), t(12)=9.10, p<.001, r^{2}=.87$, trials, and it was significantly higher than 0 in the former, $t(12)=$ 10.05, $p<.001, r^{2}=.89$, but not in the latter, $t(12)=$ $1.31, p=.219, r^{2}=.15$, condition.

As in the previous experiment, to rule out the possibility that our exclusion criterion, based solely on performance in the $2 \mathrm{AFC}$ task, may have excluded a large number of participants who had actually achieved a high performance level in the detection task, we plotted the $d$ 's of each participant in both tasks. Inspection of Fig. $3 \mathrm{~b}$ reveals that all of the excluded participants (save one, indicated by the open symbol), who showed no sensitivity in the 2AFC task, also showed no sensitivity in the target detection task.

To sum up, the second experiment had two clear results: First, we replicated the main finding of Experiment 1 , showing that awareness of the suppressed image in a

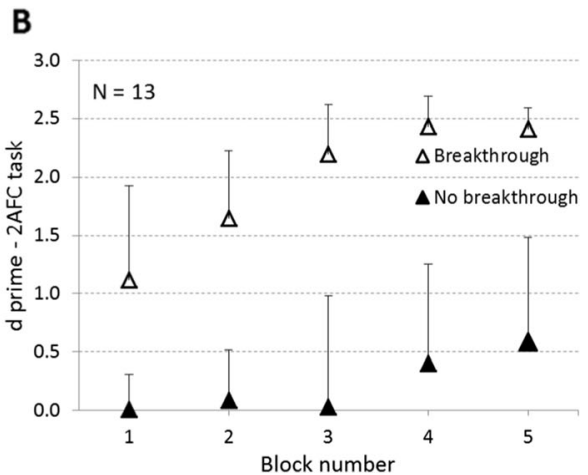

function of blocks separately for breakthrough and no-breakthrough trials. Sensitivity was significantly higher when the target was consciously perceived, as compared to when it did not break through the mask. Error bars represent $95 \%$ confidence intervals 
CFS paradigm can improve with practice. Second, we showed that the learning process underlying the improvement in awareness involved monocular retinotopic-specific information. When we changed the eye to which the target was presented during training, the level of awareness for the target dropped back to a pretraining level. However, before we should accept the conclusion that the performance drop was due to a monocular learning process in the nondominant eye, an alternative account based on visual adaptation should be considered. ${ }^{1}$

\section{Experiment 3}

Sensory adaptation, in general, refers to the fact that the response properties of neurons change as a function of their sensory history, especially when they are presented with a constant level of stimulation (Kohn, 2007). For example, the perceived contrast of a test stimulus is reduced if the visual system has been exposed (i.e., adapted) to a previous highcontrast stimulus, with stronger effects for longer adaption periods. Hence, one may note that on each trial the dominant eye received, because of the Mondrians, a stronger stimulation than the nondominant eye, to which the low-contrast target was presented. Thus, if the dominant eye was adapted to the high-contrast Mondrians during training, when the low-contrast target was presented to the same eye, the probability of target detection was strongly reduced, because the eye was adapted to a stronger contrast. The same mechanism of adaptation in the dominant eye would also explain the improved target detection and discrimination in the nondominant eye. The fact that the rate of breakthrough increased across blocks would suggest that the adaptation process was long-lasting and cumulative over time, thus progressively reducing the masking efficacy of the Mondrians.

To address this alternative account, we conducted a third, control experiment in which, first, we measured in four participants the contrast sensitivities in both eyes; second, the dominant eye was exposed to the Mondrians and, putatively, to the corresponding adaptation; third, contrast sensitivity was retested in both eyes. If the drop in performance observed on eye-switch trials in Experiment 2 had been due to sensory adaptation in the dominant eye, we would expect contrast sensitivity in this eye to be significantly and greatly reduced after prolonged exposure to the Mondrians. In the present experiment, the exposure phase was identical to that of Experiment 2 (the only exception was that we did not

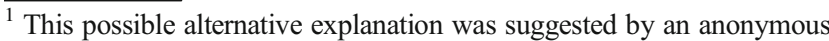
reviewer, for which we are grateful.
}

present the target in the nondominant eye), with an identical number of trials per block and the same number of blocks. In the pre- and posttest phases, we measured the contrast threshold in each eye by means of an adaptive procedure. The target consisted of a Gabor patch tilted slightly away from vertical $\left( \pm 3^{\circ}\right)$, and the participants' task was to indicate the direction of the target's orientation (left vs. right).

Method

Participants Four undergraduate students from the University of Trento (two females, two males; mean age $=27$ years) participated for course credit or monetary compensation. All participants had normal or corrected-to-normal vision and were unaware of the purpose of the study, which was carried out with the approval of the local research ethics committee. All of the volunteers gave informed consent prior to participation.

Stimuli and apparatus In the test phase, the target consisted of a Gabor patch $\left(\approx 1.5^{\circ}\right.$ in diameter). We used the following equation to represent a Gabor within a $60 \times 60$ pixel patch:

$$
F(x, y)=l_{0}\left(1+c \sin \left(2 \pi f(x \cos \theta+y \sin \theta)+\theta_{0}\right) \cdot e^{\left(-\frac{x^{2}+y^{2}}{2 \sigma^{2}}\right)}\right),
$$
with luminance $l_{0}=$ midgray, initial contrast $c=10 \%$ (then regulated by a staircase procedure; see below), spatial frequency $f=2$ cycles per degree, orientation $\theta= \pm 3^{\circ}$ from vertical (randomly determined on each trial), phase $\theta_{0}=$ $270^{\circ}$, and standard deviation of the Gaussian envelope $\sigma=$ $0.2^{\circ}$. The Gabor patch was presented against a gray background $\left(45 \mathrm{~cd} / \mathrm{m}^{2}\right)$ inside a white frame $\left(6.7^{\circ} \times 6.7^{\circ}, 0.4^{\circ}\right.$ in thickness $)$ with a central fixation dot $\left(0.2^{\circ}\right.$ diameter $)$ in the corresponding eye. The distance between the central dots of the two eyes was $9.3^{\circ}$. The mirrors of the stereoscope were adjusted so that the two images were fused into a single image, such that the presented stimuli completely overlapped retinotopically.

The exposure phase was as in Experiment 2, but the target bar was not presented in the nondominant eye.

Procedure In the test phase, on each trial the Gabor patch was presented for $100 \mathrm{~ms}$ just above the fixation point to either the dominant or the nondominant eye (randomly selected), and the participants' task was to determine whether the Gabor was tilted leftward or rightward by pressing the left-pointing arrow or the right-pointing arrow on the computer keyboard, respectively. Incorrect Gabor discriminations were signaled by the word "Error" appearing, just below the fixation point, for $500 \mathrm{~ms}$. The interstimulus interval was set at 1,000 ms.

Each test phase consisted of 400 trials, divided into two blocks of 200 trials. Within a single block, the Gabor contrast was controlled by two independent, interleaved three-down- 
one-up staircases (one for each eye), pointing at $79.4 \%$ of correct responses and consisting of 100 trials each. Each staircase used the following parameters: The initial contrast value was set at $10 \%$, and the step size varied over time $(2 \%$ for the first four reversals, $1 \%$ for the next six reversals, and $0.5 \%$ for the remaining reversals). Two new staircases were started at the beginning of each block of trials. For each eye, the mean contrast threshold was estimated by computing the arithmetic mean of the last five reversals of the corresponding staircase in a given block, and then by averaging the threshold values reached in the two blocks.

The exposure phase was like that in Experiment 2, but with two exceptions: First, the target bar was not presented to the nondominant eye. Second, no response was required of participants. Therefore, the duration of the full-contrast Mondrians presented to both eyes at the end of the trial was set at $2 \mathrm{~s}$, which corresponded to the average time required to enter both responses (yes/no and 2AFC) in Experiment 2.

\section{Results and discussion}

For each eye, the mean contrast thresholds were compared before and after exposure to the Mondrians in the dominant eye. The pre- versus posttest thresholds were not significantly different in either eye (dominant eye: pre $M=4.1 \%, S E= \pm 0.7 \%$, post $M=$ $4.5 \%, S E= \pm 0.5 \%, p=.523$; nondominant eye: pre $M=4.6 \%$, $S E= \pm 0.7 \%$, post $M=4.8 \%, S E= \pm 0.8 \%, p=.726$ ). We also analyzed the threshold changes (computed as differences) from pre- to posttest in both eyes (pre-post change in the dominant eye: $M=0.4 \%, S E= \pm 0.6 \%$, pre-post change in the nondominant eye: $M=0.1 \%, S E= \pm 0.3 \%$ ). The comparison between the two changes was not statistically significant $(p=.717$, paired $t$ test).

These results show that the presentation of the Mondrians in the dominant eye did not change its contrast sensitivity in any appreciable manner, and therefore we can safely rule out the possibility that the drop in performance observed on eyeswitch trials in Experiment 2 can be accounted for by the different levels of visual adaptation in the two eyes. In the same vein, the breakthrough improvement across blocks also cannot be explained by the same mechanism of visual adaptation in the dominant eye.

\section{General discussion}

The goal of the present study was to investigate whether, in a rivalry condition, awareness of the suppressed target image can improve with practice. To address this issue, we made use of CFS, a rivalry technique that provides an extended period of suppression for the weaker image (Tsuchiya \& Koch, 2005). In recent years, CFS has been used both to study the contribution of unconscious processing (e.g., Fang \& He, 2005; Jiang \& He, 2006) and to evaluate whether special stimuli (e.g., fearful faces) have preferential access to consciousness (see, e.g., Yang, Zald, \& Blake, 2007). When CFS is used in the latter way, participants are asked to report as quickly as possible when the suppressed image breaks through the mask, or emerges into awareness. In our study, instead of measuring the duration until breakthrough, we used the proportion of breakthrough events as an index of conscious perception of the initially suppressed image. Then, to be sure that the reported breakthrough events corresponded to genuine occurrences of conscious perception, we addressed participants' sensitivity to the target location in a 2 AFC task. In both experiments, the proportions of breakthrough events significantly increased across blocks, showing that in the present CFS paradigm, awareness of the weaker target image improved with training. Furthermore, the results of the last block, on which after training we changed either the target orientation (Exp. 1) or the eye to which the target was presented (Exp. 2), indicated that the learning process leading to the improvement in target awareness occurred primarily in monocular retinotopic-specific neural representations beyond those specific to target orientation. Our results are, overall, in line with those of previous studies that had shown that practice can affect conscious perceptual experience during rivalry conditions (e.g., Ludwig et al., 2013; Paffen et al., 2008).

What possible mechanisms could account for an increasing tendency for breakthrough of an initially suppressed target image? A first possible candidate is increases in the local, unconsciously specified target salience over time, enhancing the target's likelihood of breaking through the flickering mask. Jiang, Costello, Fang, Huang, and He (2006) have shown that unconsciously defined salience can increase at the location occupied by the invisible stimuli presented in the nondominant eye during CFS. Although the highly salient motivational stimuli (erotic pictures) used in this previous study cannot be compared to the simple vertical/horizontal gray bars used here, we cannot exclude the possibility that in a similar fashion, changes in unconsciously specified target salience might explain our results.

A second possibility is decreasing salience of the flickering mask because of visual adaptation to either the contrast or the motion signals associated with the Mondrians in the dominant eye. According to this explanation, adaptation was built up across blocks in the dominant eye, thus resulting both in facilitation of target breakthrough for the nondominant eye during training and in the dramatic drop in breakthrough events when the target was presented to the untrained dominant eye. However, although local breakthrough events can be triggered during binocular rivalry by a process associated with neural adaptation (Alais, Cass, O'Shea, \& Blake, 2010; van Ee, 2009, 2011), the results of Experiment 3 rule out the 
possibility that in the present CFS paradigm, adaptation to the Mondrians in the dominant eye could account for our main findings.

A third possibility involves changes in target salience due to exogenous attention. According to this view, exogenous attention might have been attracted to the unconscious monocular target representation, thus increasing its salience and favoring further breakthrough. In line with this possibility, recent studies have shown that exogenous attention can be summoned by monocular supraliminal onsets in a spatialcueing paradigm (Self \& Roelfsema, 2010). In addition, and even more relevant to the present hypothesis, it has been found that attention (and eye movements) can also be unconsciously shifted toward a suppressed target singleton during CFS (Hsieh, Colas, \& Kanwisher, 2011; Rothkirch, Stein, Sekutowicz, \& Sterzer, 2012; Zhaoping, 2008).

A fourth possibility involves changes in target salience due to top-down (volitional) or endogenous attention, which - as was originally observed by von Helmholtz (1866/1925) - has been shown to influence binocular rivalry (Ooi \& He, 1999), and is also important for other forms of learning in visual perception, as in the case of perceptual learning (Fahle \& Poggio, 2002). There are two ways in which this form of attention could have favored the learning process in our CFS paradigm. One possibility is that voluntary attention operated directly on the suppressed target image, progressively increasing its chances of breaking through the mask. Two considerations seem to undermine the feasibility of this putative mechanism: First, it has been reasonably argued and shown that one cannot voluntary direct attention to an invisible stimulus (Schall, Nawrot, Blake, \& Yu, 1993), and accordingly, binocular rivalry studies have indicated that voluntary attention seems incapable of operating on the neural representation of the suppressed image (Chong et al., 2005). Second, there is evidence that in a dichoptic viewing condition-typical of binocular rivalry and CFS - the observer cannot tell which eye is being stimulated by a given stimulus (Ono \& Barbeito, 1985; Wolfe \& Franzel, 1988). A second possibility is that in our CFS paradigm, initially, on some trials, the suppressed target image broke through the mask because of the stochastic nature of rivalry. If this happened, it could be that voluntary attention was allocated to the visible target to perform the task. However, our results showed that attention did not enhance the stimulus-specific representations, but rather the neural representation of the eye to which the target was presented. Hence, as was suggested by Ooi and He (1999), we could speculate that top-down attentional signals reached early monocular representations of the target at the corresponding retinotopic locations, thus increasing its strength, and making the boosted monocular, retinotopic-specific representations more likely to break through on successive trials. According to this account, top-down attention would alter the target representation only after it became conscious (Chong et al.,
2005). Thus, as the experiment unfolded, the monocular representation of the target, competing with that of the mask, would have become progressively more salient, and therefore more likely to break through and be consciously perceived.

Possible candidate areas where attention (either exogenous or endogenous) might have changed the strength of a monocular representation of the target over the course of training include the ocular dominance columns of V1 (although the contribution of V1 binocular neurons with strong monocular preference cannot be excluded) and the lateral geniculate nucleus, two structures in which the information from the two eyes remains segregated and in which attentional effects have been documented (O'Connor, Fukui, Pinsk, \& Kaster, 2002). Interestingly, both brain areas have also been found to show reliable modulation under binocular rivalry, such that neural activations corresponding to one eye were larger when the observer was aware of the image presented to that eye (Tong et al., 2006). That voluntary attention may affect perception at early (monocular) levels of visual analysis during interocular competition has also been suggested by Klink, van Ee, Nijs, Brouwer, Noest, and van Wezel (2008). The authors used intermittent presentations of bistable visual stimuli (e.g., binocular rivalry) to study the brain mechanisms controlling which of the two competing perceptual interpretations first reaches visual awareness. Their results demonstrated that although rivalry is largely determined by low-level adaptation mechanisms, participants showed a good degree of voluntary control over the rivalry condition, which suggests that topdown attention can reach early stages of sensory processing.

Finally, we consider the implications of our results with respect to the phenomenon of sensory eye dominance (SED). SED consists of the fact that in normal binocular vision, one eye contributes more than the other to the combined binocular view, a condition that is thought to arise from unbalanced interocular inhibition (Ooi \& He, 2001). Our results, showing that monocular information can be used to increase the likelihood of awareness of the weaker stimulus during $\mathrm{CFS}$, are in agreement with those reported by $\mathrm{Xu}, \mathrm{He}$, and Ooi (2010; see also Xu, He, \& Ooi, 2012b). These authors showed that, by means of appropriate training combining attentional cueing, perceptual learning, and binocular rivalry, SED can be reduced. Specifically, they devised an innovative push-pull training protocol, in which a pair of dichoptic orthogonal gratings are simultaneously presented to the two eyes. However, before presentation of the gratings, a transient cue captures exogenous attention in the nondominant eye. As a result, the corresponding gratings image wins the rivalry with the dominant eye (by means of interocular inhibition). When participants perform a discriminative task on the perceived target grating, the results reveal that when this protocol was administered for several days, SED was considerably reduced (Xu et al., 2010), indicating that training modified the strengths of the eye-specific neural representations, as well 
as their contributions to the binocular view. Interestingly, a recent study has shown that this protocol can be used to reduce amblyopia, an extreme and pathological case of SED (Ooi, $\mathrm{Su}$, Natale, \& He, 2013).

If we consider that SED is measured by evaluating which eye-specific information wins the competition and is consciously perceived in a binocular rivalry condition, then we can conclude that our study, based on CFS, and those of $\mathrm{Xu}$, He, and Ooi (e.g., 2010, 2012b), based on binocular rivalry, concur in showing that awareness of eye-specific neural representations can be improved with practice. Indeed, whereas in our study, practice increases the probability for the weak image to break through the mask to become conscious, in $\mathrm{Xu}$, $\mathrm{He}$, and Ooi's push-pull paradigm, training reduces the level of image intensity required from the nondominant eye that is needed to overcome the dominance of the dominant eye's image during binocular rivalry. As a further link between the two methods, we note that the push-pull idea might have been implicitly present in our CFS paradigm, in that perceptual training in one eye was continuously paired with strong stimulation (the dynamic CFS mask) in the other eye. Furthermore, although Xu, He, and Ooi (2010, 2012a, 2012b) used binocular rivalry to assess SED, SED can also be measured by using CFS (Yang, Blake, \& McDonald 2010).

Yet some differences exist between the two paradigms. First, although only a direct comparison between the two methods could provide a definitive answer, it would seem that our paradigm could be faster in inducing changes in the monocular representation. We found a significant increase in the probability of target breakthrough with a relatively modest number of trials in a single training session, whereas the push-pull training protocol is administered for several days (Xu et al., 2010). In addition, the drop in breakthrough events for the untrained eye that we observed in Experiment 2 on eye-switch trials seems to indicate that our paradigm was able not only to locally reduce SED, but also to locally reverse it after a limited amount of practice. However, because we did not get any precise measures of SED before and after training, and because we did not know how much SED changes could generalize to other retinal locations or stimulus features, we believe that more work will be needed to fully explore the degree to which our CFS-based paradigm can affect SED. Second, the monocular improvement leading to SED reduction, and induced by means of the push-pull protocol, appears to be quite specific to the stimulus properties used during training. For example, the reduction of SED obtained with either vertical or horizontal training gratings does not generalize to other gratings with oblique orientations. Analogously, the beneficial effect of training on SED observed for a given retinal location does not transfer much to a different location (Xu et al., 2010). This high degree of spatial and orientation specificity, which is a hallmark of perceptual learning, was interpreted by Xu, He, and Ooi (2010) as evidence that early visual cortex, where a high degree of specificity is retained, is the most likely candidate for the neural changes underlying the reduction in SED. By using both vertical and horizontal orientations during training, the same authors were able to generalize the SED reductions across different oblique orientations, thus improving the impact of their training protocol (Xu, He, \& Ooi, 2012a). However, it should be noted that in our CFS paradigm we found evidence of a monocular learning process that largely and spontaneously generalized across orthogonal stimulus orientations, despite the fact that during training we had presented a stimulus with a fixed orientation (either vertical or horizontal). Third, one of the key ingredients of the push-pull training protocol to obtaining enhanced processing of the input to the nondominant eye is that attention must be exogenously attracted to the corresponding image by virtue of an abrupt visual onset (Xu et al., 2012a, 2012b). By contrast, our paradigm was not specifically designed to favor an exogenous attention shift to the target, although, as we discussed above, we cannot rule this possibility out. In our view, if our results are driven by attention, the present CFS-based paradigm is more likely to affect SED by means of top-down endogenous attention. For example, if the target was, say, horizontal, when it broke through, the observer might over time develop an expectation for a horizontal target, which could feed back to the early monocular retinotopic representations, enhancing the expected orientation, or perhaps inhibiting the orthogonal orientation. One possible neural mechanism of selective attention that affords top-down modulation of sensory processing has recently been described (Zhang et al., 2014). Because this mechanism involves the modulation of local retinotopic circuitry, this mechanism would not be orientation-specific in its enhancements, but only retinotopically specific, consistent with our finding that improvements generalize to new orientations. Our findings suggest, moreover, that such a mechanism must feed back to a monocular level of representation, whether in V1 or the LGN.

In conclusion, we have documented a rapid perceptuallearning phenomenon that occurs over monocular retinotopic-specific information but that generalizes over changes in stimulus orientation. This rules out perceptual learning in specific classes of orientation-tuned cells, such as simple cells in V1, and also rules out perceptual learning at a later stage, after which information about the eye of origin has been lost. This effect could be mediated by bottom-up and unconscious changes in stimulus salience, adaptation of the CFS mask, or attention-induced increases in salience with learning that could in principle operate as early as the thalamus, which might bias interocular competition at early or later stages of visual analysis. Our results also suggest that CFS could be used as an efficient 
tool to change SED, which can have important implications for the treatment of pathological SED conditions such as amblyopia.

Author note All authors developed the study concept and contributed to the study design. The testing and data collection were performed by T.M., who also performed the data analysis and interpretation under the supervision of P.U.T. and M.T. T.M. and M.T. drafted the manuscript, and P.U.T. provided critical revisions. All of the authors approved the final version of the manuscript for submission.

\section{References}

Alais, D., Cass, J., O’Shea, R. P., \& Blake, R. (2010). Visual sensitivity underlying changes in visual consciousness. Current Biology, 20, 1362-1367.

Almeida, J., Mahon, B. Z., \& Caramazza, A. (2010). The role of the dorsal visual processing stream in tool identification. Psychological Science, 21, 772-778. doi:10.1177/0956797610371343

Blake, R. (1989). A neural theory of binocular rivalry. Psychological Review, 96, 145-167. doi:10.1037/0033-295X.96.1.145

Blake, R., \& Logothetis, N. K. (2002). Visual competition. Nature Reviews Neuroscience, 3, 13-21. doi:10.1038/nrn701

Brainard, D. H. (1997). The Psychophysics Toolbox. Spatial Vision, 10, 433-436. doi:10.1163/156856897X00357

Chong, S. C., Tadin, D., \& Blake, R. (2005). Endogenous attention prolongs dominance durations in binocular rivalry. Journal of Vision, 5 , 1004-1012. doi:10.1167/5.11.6

Fahle, M., \& Poggio, T. (2002). Perceptual learning. Cambridge, MA: MIT Press.

Fang, F., \& He, S. (2005). Cortical responses to invisible objects in the human dorsal and ventral pathways. Nature Neuroscience, 8,1380 1385 .

Fogelson, S. V., Kohler, P. J., Miller, K. J., Granger, R., \& Tse, P. U. (2014). Unconscious neural processing differs with method used to render stimuli invisible. Frontiers in Psychology, 5, 601. doi:10. 3389/fpsyg.2014.00601

Frank, S. M., Reavis, E. A., Tse, P. U., \& Greenlee, M. W. (2014). Neural mechanisms of feature conjunction learning: Enduring changes in occipital cortex after a week of training. Human Brain Mapping, 35, 1201-1211. doi:10.1002/hbm.22245

Freeman, A. W. (2005). Multistage model for binocular rivalry. Journal of Neurophysiology, 94, 4412-4420.

Green, D. M., \& Swets, J. A. (1966). Signal detection theory and psychophysics. New York, NY: Wiley.

Hsieh, P. J., Colas, J. T., \& Kanwisher, N. (2011). Pop-out without awareness: Unseen feature singletons capture attention only when topdown attention is available. Psychological Science, 22, 12201226. doi: $10.1177 / 0956797611419302$

Hubel, D. H., \& Wiesel, T. N. (1977). Functional architecture of macaque monkey visual cortex. Proceedings of the Royal Society B, 198, 159.

Jiang, Y., Costello, P., Fang, F., Huang, M., \& He, S. (2006). A genderand sexual orientation-dependent spatial attentional effect of invisible images. Proceedings of the National Academy of Sciences, 103, 17048-17052.

Jiang, Y., Costello, P., \& He, S. (2007). Processing of invisible stimuli: Advantage of upright faces and recognizable words in overcoming interocular suppression. Psychological Science, 18, 349-355. doi: 10.1111/j.1467-9280.2007.01902.x
Jiang, Y., \& He, S. (2006). Cortical responses to invisible faces: Dissociating subsystems for facial-information processing. Current Biology, 16, 2023-2029. doi:10.1016/j.cub.2006.08.084

Kim, C. Y., \& Blake, R. (2005). Psychophysical magic: Rendering the visible "invisible.". Trends in Cognitive Sciences, 9, 381-388.

Klink, P. C., van Ee, R., Nijs, M. M., Brouwer, G. J., Noest, A. J., \& van Wezel, R. J. A. (2008). Early interactions between neuronal adaptation and voluntary control determine perceptual choices in bistable vision. Journal of Vision, 8(5), 16:1-18. doi:10.1167/8.5.16

Kohn, A. (2007). Visual adaptation: Physiology, mechanisms, and functional benefits. Journal of Neurophysiology, 97, 3155-3164.

Kovács, I., Papathomas, T. V., Yang, M., \& Fehér, A. (1996). When the brain changes its mind: Interocular grouping during binocular rivalry. Proceeding of the National Academy of Sciences, 93, 1550815511.

Leopold, D. A., \& Logothetis, N. K. (1996). Activity changes in early visual cortex reflect monkeys' percepts during binocular rivalry. Nature, 379, 549-553.

Logothetis, N. K., Leopold, D. A., \& Sheinberg, D. L. (1996). What is rivalling during binocular rivalry? Nature, 380, 621-624.

Ludwig, K., Sterzer, P., Kathmann, N., Franz, V. H., \& Hesselmann, G. (2013). Learning to detect but not to grasp suppressed visual stimuli. Neuropsychologia, 51, 2930-2938.

Meng, M., \& Tong, F. (2004). Can attention selectively bias bistable perception? Differences between binocular rivalry and ambiguous figures. Journal of Vision, 4(7), 539-551. doi:10.1167/4.7.2

O'Connor, D. H., Fukui, M. M., Pinsk, M. A., \& Kaster, S. (2002). Attention modulates responses in the human lateral geniculate nucleus. Nature Neuroscience, 5, 1203-1209.

Ono, H., \& Barbeito, R. (1985). Utrocular discrimination is not sufficient for utrocular identification. Vision Research, 25, 289-299.

Ooi, T. L., \& He, Z. J. (1999). Binocular rivalry and visual awareness: The role of attention. Perception, 28, 551-574. doi:10.1068/p2923

Ooi, T. L., \& He, Z. J. (2001). Sensory eye dominance. Journal of American Optometry Association, 72, 168-178.

Ooi, T. L., Su, Y. R., Natale, D. M., \& He, Z. J. (2013). A push-pull treatment for strengthening the "lazy eye" in amblyopia. Current Biology, 23, R309-R310. doi:10.1016/j.cub.2013.03.004

Paffen, C. L. E., Verstraten, F. A. J., \& Vidnyànszky, Z. (2008). Attentionbased perceptual learning increases binocular suppression of irrelevant visual features. Journal of Vision, 8(4), 25:1-11. doi:10.1167/8. 4.25

Rothkirch, M., Stein, T., Sekutowicz, M., \& Sterzer, P. (2012). A direct oculomotor correlate of unconscious visual processing. Current Biology, 22, R514-R515. doi:10.1016/j.cub.2012.04.046

Sasaki, H., \& Gyoba, J. (2002). Selective attention to stimulus features modulates interocular suppression. Perception, 31, 409-419.

Schall, J., Nawrot, M., Blake, R., \& Yu, K. (1993). Visually guided attention is neutralized when informative cues are visible but unperceived. Vision Research, 33, 2057-2064.

Self, M. W., \& Roelfsema, P. R. (2010). A monocular, unconscious form of visual attention. Journal of Vision, 10(4), 17:1-22. doi:10.1167/ 10.4.17

Tong, F., Meng, M., \& Blake, R. (2006). Neural bases of binocular rivalry. Trends in Cognitive Sciences, 10, 502-511. doi:10.1016/j.tics. 2006.09.003

Tsuchiya, N., \& Koch, C. (2005). Continuous flash suppression reduces negative afterimages. Nature Neuroscience, 8, 1096-1101.

Tsuchiya, N., Koch, C., Gilroy, L. A., \& Blake, R. (2006). Depth of interocular suppression associated with continuous flash suppression, flash suppression, and binocular rivalry. Journal of Vision, 6(10), 6:1068-1078. doi:10.1167/6.10.6

van Ee, R. (2009). Stochastic variations in sensory awareness are driven by noisy neuronal adaptation: Evidence from serial correlations in perceptual bistability. Journal of the Optical Society of America A, 26, 2612-2622. 
van Ee, R. (2011). Percept-switch nucleation in binocular rivalry reveals local adaptation characteristics of early visual processing. Journal of Vision, 11(2), 13:1-12. doi:10.1167/11.2.13

von Helmholtz, H. (1925). Treatise on physiological optics (Vol. III, J. P. C. Southall, Ed. \& Trans.). New York, NY: Dover. (Original work published 1866).

Wheatstone, C. (1838). On some remarkable, and hitherto unobserved, phenomena of binocular vision. Philosophical Transactions of the Royal Society, 128, 371-394.

Wilson, H. R. (2003). Computational evidence for a rivalry hierarchy in vision. Proceedings of the National Academy of Sciences, 100, 14499-14503.

Wolfe, J. M., \& Franzel, S. L. (1988). Binocularity and visual search. Perception \& Psychophysics, 44, 81-93. doi:10.3758/BF03207480

Xu, J. P., He, Z. J., \& Ooi, T. L. (2010). Effectively reducing sensory eye dominance with a push-pull perceptual learning protocol. Current Biology, 20, 1864-1868. doi:10.1016/j.cub.2010.09.043

Xu, J. P., He, Z. J., \& Ooi, T. L. (2012a). Perceptual learning to reduce sensory eye dominance beyond the focus of top-down visual attention. Vision Research, 61, 39-47. doi:10.1016/j.visres.2011.05.013
Xu, J. P., He, Z. J., \& Ooi, T. L. (2012b). Push-pull training reduces foveal sensory eye dominance within the early visual channels. Vision Research, 61, 48-59. doi:10.1016/j.visres. 2011.06.005

Yang, E., Blake, R., \& McDonald, J. E., II. (2010). A new interocular suppression technique for measuring sensory eye dominance. Investigative Ophthalmology and Visual Science, 51, 588-593. doi: $10.1167 /$ iovs.08-3076

Yang, E., Zald, D. H., \& Blake, R. (2007). Fearful expressions gain preferential access to awareness during continuous flash suppression. Emotion, 7, 882-886. doi:10.1037/1528-3542.7. 4.882

Zhang, S., Xu, M., Kamigaki, T., Hoang, D. J. P., Chang, W. C., Jenvay, S., \& Dan, Y. (2014). Selective attention: Long-range and local circuits for top-down modulation of visual cortex processing. Science, 345, 660-665. doi:10.1126/science. 1254126

Zhaoping, L. (2008). Attention capture by eye of origin singletons even without awareness - A hallmark of a bottom-up saliency map in the primary visual cortex. Journal of Vision, 8(5), 1:1-18. doi:10.1167/8.5.1 